\title{
Cerebral Toxoplasmosis in HIV - A diagnostic challenge
}

\author{
Serasinghe $\mathrm{PS}^{1}$, Alahakoon $\mathrm{B}^{1}$, Weerasooriya $\mathrm{N}^{1}$, Jayanaga $\mathrm{A}^{1}$
}

\section{Introduction}

Focal neurological disease in patients with acquired immunodeficiency syndrome may be caused by various opportunistic pathogens and malignancies, including Toxoplasma gondii, progressive multifocal leukoencephalopathy (PML), and primary central nervous system lymphoma (PCNSL). Diagnosis may be difficult, because the findings of lumbar puncture, computed tomography (CT), and magnetic resonance imaging (MRI) are relatively nonspecific with a varying degree of overlap.

Toxoplasmosis is the most common central nervous system infection in patients with the acquired immunodeficiency syndrome (AIDS). ${ }^{1}$ Latent infectionwith primary toxoplasmosis in an immunocompetenthost is usually asymptomatic andcan persist for life. In immunosuppressed patients, the disease can reactivate when the CD4 count falls below 100 cells/microL. ${ }^{2}$ Primary CNS lymphoma is the second most common cause of focal brain disease in patients with AIDS with similar symptomatology to cerebral toxoplasmosis. 3

We present a case of cerebral toxoplasmosis in a HIV infected patient with a discussion on the difficulties we come across during the diagnosis. sexual practices. On examination patient had oral thrush, bilateralhorizontal nystagmus and exaggerated reflexes in all 4 limbs. HIV infection wasconfirmed with western blot and CD4 count was46cells/microL. Full blood count showed absolute lymphocyte count of0.67x109/L. MRI of brain showed multiple, intermediate signal intensity focal lesionsof varying sizes with ring enhancement scattered throughout the cerebralhemispheres, brainstem and basal ganglia.

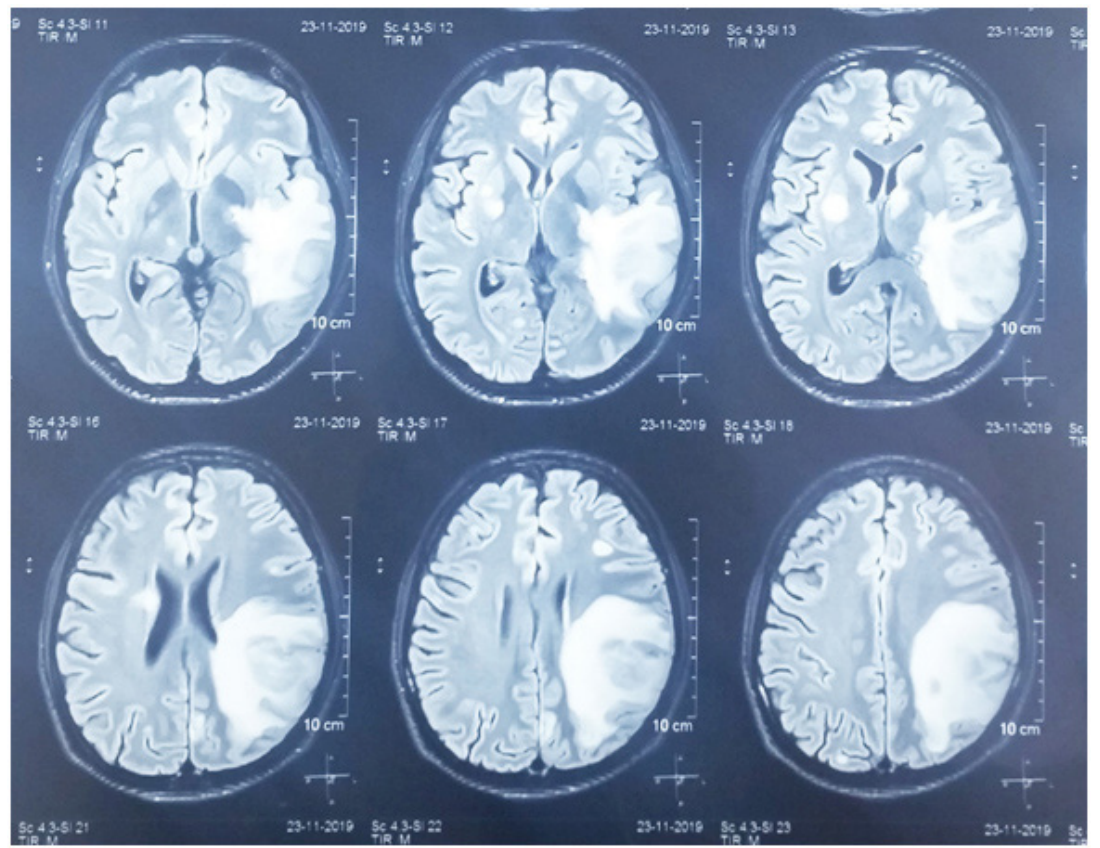

Figure 1 - T1 weighted axial MRI brain shows multiple focal lesions with ring enhancement

\section{Case Report}

A 24-year-old man presented with progressively worseningheadache for 3 weeks duration. He did not have fever. Two weeks into the illness he developed on and off blurring ofvision. At the same time his family noticed a change of his personality, an impaired memory, slowness of thought and dull sensorium. On the day of admission to the hospital he developed a generalized tonic-clonicseizure. He has had multiple sexual partners of both sexes,andhad unsafe

1. National Hospital, Sri Lanka

Corresponding Author:

Pasan Serasinghe

E mail: pasansanda@gmail.com

iD https://orcid.org/0000-0001-8766-4018
Largest lesion measured $3.3 \mathrm{~cm} \times 3.0 \mathrm{~cm}$ in maximum axial dimensions in the left parietal lobe with marked peri-lesionaloedema. Further investigations revealed positive toxoplasma IgG antibodies. Cerebrospinal fluid (CSF) analysis was not done as lumbar puncture was not attempted in the presence of focal neurology. Patient made a remarkable clinical recovery with empirical toxoplasmosis treatment with sulfadiazine and pyrimethamine along with leucovorin. His mental state and neurological deficit improved. He was started on antiretroviral therapy 2 weeks after initiating toxoplasmosis treatment. Follow up MRI scan with contrast performed after 4 weeks of initiation of treatment showed resolving lesions. Brain biopsy was deferred as patient was well responding to toxo- 
plasmosis treatment.

\section{Discussion}

Toxoplasmosis, caused by protozoan parasite, Toxoplasma gondii, is one of the most common CNS infections in HIV/AIDS patients. The susceptibility to toxoplasmosis increases in those who are not on prophylaxis nor on antiretroviral therapy. Typically, patients with toxoplasma encephalitis present with headache, confusion, fever, focal neurological deficits, mental status changes, seizures or coma. ${ }^{1}$ The typical CT and MRI findings in patients with toxoplasmosis are more than 2 ring-enhancing lesions with surrounding edema.Definitive diagnosis requires detection of protozoa in brain biopsy or detection of T.gondii by polymerase chain reaction (PCR) in CSF. Primary CNS lymphoma should be considered in a patient with HIV infection who presents with focal neurology.4In $4 \%-7 \%$ of Human Immune deficiency Virus (HIV) infected patients with neurological complaints had primary CNS lymphoma diagnosed. ${ }^{4}$ Primary CNS lymphoma is a late manifestation of HIV that occurs when the CD4 cell count drops below 50 cells/microL. The clinical presentation of cerebral toxoplasmosis is very much similar to that of PCNSL. The presenting signs and symptoms of primary CNS lymphoma include headache, altered mental status, memory loss, lethargy, hemiparesis and seizures. ${ }^{5}$ The PCNSL has been designated an acquired immune deficiency syndrome defining disease.

CSF analysis would be useful to differentiate PCNSL from cerebral toxoplasmosis. PCR for T.gondii in CSF has a specificity approaching $96-100 \%$. The EpsteinBarr virus (EBV) genome can be found in lymphoma cells in nearly $100 \%$ of cases of AIDS-related primary CNS lymphoma. ${ }^{6}$ The presence of EBV DNA in the cerebrospinal fluid supports the diagnosis of PCNSL with a specificity ranging from $94 \%$ to $100 \%$.In our patient focal neurology precluded CSF analysis.

In the absence of serology and CSF analysis neuroimaging studies are vital in the differential diagnosis. However there is a considerable overlap exist in the findings of neuroimaging in these two conditions.In toxoplasmosis MRI is the preferred imaging modality. Primary CNS lymphoma frequently give rise to a solitary lesion, whereas toxoplasmosis is usually multifocal and PCNSL typically demonstrates sub ependymal spread, whereas toxoplasmosis tends to be scattered through the basal ganglia and at the corticomedullary junction. 7,8 Typicallylesions in toxoplasma encephalitis show ring or nodular enhancement with contrast. However, lesions of PCNSL on neuroimaging show a homogenous contrast en- hancement. In both conditions lesions exhibit mass effect with surrounding edema. As in cerebral toxoplasmosis, PCNSL lesions too may sometimes show ring enhancement. ${ }^{7}$ Thus, the pattern of enhancement may not be helpful to differentiate.

Toxoplasmosis and lymphoma have significant overlap in terms of diffusion characteristics too. Toxoplasmosis tends to have more facilitated diffusion while lymphoma tends to have more restricted diffusion. In this patient diagnosis of toxoplasma encephalitis is madebased on clinical presentation, brain imaging and positive serology. Multiple ring enhancing lesions on imaging and toxoplasma serology was in favor of the diagnosis.Brain biopsy and directly visualizing the tachyzoites or cyst provides a definitive diagnosis. ${ }^{9}$ Brain biopsy would have ruled out the possibility of PCNSL. However when the lesions are surgically inaccessible conservative approach is preferred. Treatment should be monitored with serial neuro imaging. The clinical improvement should be parallel with radiological resolution of the lesions. Failure to improve symptoms clinically and worsening or persistence of radiological lesions within 2 weeks should raise concern for alternative diagnosis and a brain biopsy should be considered. Our patient responded well to empirical toxoplasma treatment and repeat MRI scan confirmed radiological improvement in previously documented lesions. Therefore brain biopsy was not considered. This case highlights the difficulties one would face when differentiating cerebral toxoplasmosis from PCNSL in HIV patients. Experienced radiological services are essential in making the correct diagnosis.

\section{References}

1.Luft B, Remington J. AIDS commentary: toxoplasmic encephalitis. J Infect Dis 1988; 157:1-6

2. Porter S, Sande M. Toxoplasmosis of the central nervous system in the acquired immunodeficiency syndrome. N Engl J Med 1992; 327: 1643-8

3.Skiest D J. Focal Neurological Disease in Patients with Acquired Immunodeficiency Syndrome. Clinical Infectious Disease 2002;34:1:103-115,

4. Ciacci JD, Tellez C, VonRoenn J, Levy RM. Lymphoma of the central nervous system in AIDS. Semin Neurol 1999; 19:213-21

5. Fine HA, Mayer RJ. Primary central nervous system lymphoma. Ann Intern Med 1993; 119:1093-104.

6. Cinque $P$, Vago L, Dahl $H$, et al. Polymerase chain reaction on cerebrospinal fluid for diagnosis of virus-associated opportunistic diseases of the central nervous system in HIV-infected patients. AIDS 1996; 10:951-8

7.Walot I, Miller B, Chang L, Mehringer C. Neuroimaging findings in patients with AIDS. Clin Infect Dis 